\title{
Developments in Plant-Based Vaccines Against Diseases of Concern in Developing Countries
}

\author{
Evangelina Gómez, Silvina Chimeno Zoth, Elisa Carrillo and Analía Berinstein*
}

\author{
Instituto de Biotecnología, CICVyA, INTA, Castelar, CC 25 B1712WAA, Buenos Aires, Argentina \\ Consejo Nacional de Investigaciones Cientificas y Técnicas (CONICET), Rivadavia 1917 C1033AAJ, Ciudad de Buenos \\ Aires, Argentina
}

\begin{abstract}
Vaccination has been extensively used to prevent, eradicate and control infectious diseases. However, the development and implementation of new vaccines remains prohibitively expensive for middle and low income countries where such measures are surely needed most. The production of plant-based vaccines provides a promising alternative to create affordable biological products. In the past decade, a growing number of research groups worldwide have studied plant expression and parenteral or oral delivery of vaccine antigens, some showing promising potential. Here, we describe the basis of the system and the state of the art of developments regarding diseases of concern in developing countries.
\end{abstract}

Keywords: Human pathogens, plant-based vaccines, transgenic plants, developing countries, oral delivery, subunit vaccines, antibody response.

\section{INTRODUCTION}

Since the pioneering experiments of immunization conducted by Edward Jenner in 1976 to control smallpox, vaccination has been extensively used to prevent, eradicate and control infectious diseases. Vaccines, designed to protect against diseases by inducing specific immunity, are extensively and routinely administered around the world based on the principle that it is always better to maintain people healthy than to treat them once they are ill. Immunization is a proven tool for controlling and even eradicating diseases. One of the best examples is the immunization campaign carried out by the World Health Organization (WHO) from 1967 to 1977 to eradicate the natural occurrence of smallpox. When the program began, the disease still threatened $60 \%$ of the world's population and killed every fourth victim. Polio is also a well known example; since the launch by WHO and its partners of the Global Polio Eradication Initiative in 1988, infections have fallen by $99 \%$, and nearly five million people have escaped paralysis. Another example is the fight against measles; between 1999 and 2003, measles deaths dropped worldwide by almost $40 \%$ and some regions have set a target of eliminating the disease (www.who.int).

As stated by the Pan American Health Organization (PAHO), Countries of the Americas have led the way in vaccine-preventable disease eradication (smallpox and poliomyelitis), elimination (endemic transmission of measles), and control (pertussis, diphtheria, tetanus, invasive diseases caused by Haemophilus influenzae type b, and hepatitis B). These achievements have been secured by accomplishing and maintaining high levels of immunization coverage, accomplishing

*Address correspondence to this author at the Instituto de Biotecnología, CICVyA, INTA, Castelar, CC 25 B1712WAA, Buenos Aires, Argentina; Tel: 54-11-4621-1278; Fax: 54-11-4621-0199,

E-mail: aberinstein@cnia.inta.gov.ar effective surveillance, and conducting mass immunization campaigns. However, even when significant improvements have been made towards implementing essential immunization practices in developing countries, the complexities of developing new vaccines and the obstacles faced in bringing them to vulnerable populations demand continued action. Numerous new vaccines with interesting potential for improving health in the most needed part of the world are in the research and development pipeline. They include vaccines for rotavirus diarrhea, human papillomavirus, and pneumococcal disease. However, due to limitations in the availability and uses of interventions that proved efficient at community levels, pneumonia and diarrhea still kill almost 4 millions of children under the age of 5 every year.

The majority of animal pathogens initiate disease following interaction with the mucosal surfaces lining the digestive, respiratory or genital tracts [1-3]. The primary defense of these tissues is the mucosal immune system. A mucosal immune response begins with the recognition of the pathogen by specialized cells in the epithelium that overlay lymphoid follicles and it is characterized by the production of secretory immunoglobulin (Ig) type A. Parenteral vaccinedelivery induces serum antibody responses and only rarely elicits IgA responses [4]. In contrast, oral or nasal vaccinedelivery is more effective at stimulating mucosal immunity [5, 6]; thus, mucosal vaccination is thought to be an ideal strategy for combating both emerging and reemerging infectious diseases $[7,8]$.

For the implementation of a successful global vaccination strategy, a well designed subunit oral vaccine system should satisfy, at least, the following criteria: (a) produce sufficient quantities of desired antigen [9], $(b)$ preserve the expressed antigen for a long time at room temperature $[10,11],(c)$ induce protective immunity $[6,11]$, and $(d)$ be protected from enzymatic digestion in the gastrointestinal tract [6]. 
Development of edible vaccines based on viral or bacterial subunits could be an interesting tool to induce protection at the portal entrance for many pathogens. For different reasons, the design of efficient edible vaccines by means of transgenic plants represents a challenging alternative to the conventional ones [12]. The simplicity of their production, handling and administration makes them an attractive option for developing affordable vaccines. In addition, products from transgenic plants are unlikely to be contaminated by animal pathogens, microbial toxins or oncogenic sequences $[13,14]$. The use of transgenic plants for the expression of different antigens has been increasingly employed for the production of experimental immunogens. Several authors have reported antibody response to parenterally or orally administered plantderived antigens [15-21]. The demonstration that many proteins from pathogens, including those expressed in transgenic plants, are immunogenic when administered orally, has encouraged the study of other antigens expressed in plants with the goal of developing edible vaccines. We described here the basis of the system and the state of the art of developments regarding diseases of concern in developing countries.

\section{PLANTS AS BIOREACTORS}

Plants have emerged not only as an interesting alternative for the production of a broad range of heterologous molecules but as a tool for oral delivery as well. The notion of a plantbased vaccine that could be eaten to stimulate a mucosal immune response was an auspicious idea that continues to be investigated.

Heterologous protein expression in plants can be achieved by several transformation techniques regarding the plant species or the type of tissue that will be transformed. The delivery of the foreign DNA into the plant cell can be performed by (a) direct gene transfer methods as polyetilenglicol treatment or electroporation; $(b)$ by using a carrier like in microinjection or biolistics; and (c) by employing biological vectors. The preferable method of choice, when possible, is the use of the biological vector Agrobacterium, a soil pathogenic bacterium that naturally infects and transfers its virulent genes to the plant cell nucleus. The selected gene of interest is first introduced into Agrobacterium and the method makes profit of the bacteria's capability of introducing, into the plant, large segments of DNA with minimal rearrangement at high efficiencies and at a low cost. Once the foreign DNA is in the nucleus, the integration occurs randomly by non-homologous recombination. After several rounds of cell growth and regeneration in selective media, this process results in stably transformed plants. A wide range of crop species have been used for the heterologous expression of proteins such as tobacco, Arabidopsis or alfalfa, including edible species like tomato, potato, rice, lettuce, banana or maize, with the purpose of attaining an edible vaccine. The intrinsic features of plants as eukaryotic expression systems have made possible the expression of multi-component proteins and molecules with complex structures. An example of these properties is the capability of plants to express, process and assemble full length and single-chain antibodies $(\mathrm{scFv})$ retaining full biological activities [22-25].
Due to the low yields usually recovered from transgenic lines and the time-consuming the developing and characterization of a transgenic plant is, many strategies have been conceived in order to optimize the expression system. One example is the introduction of the foreign DNA into the chloroplast genome getting profit of its high number of copies per cell and transcriptional rate as well as the lack of problems associated with positional effects. Using this approach, a yield as high as $47 \%$ of the total soluble proteins (PTS) in leaves can be reached [26].

Heterologous expression in plants can also be performed by transient transformation, where the foreign DNA is not integrated but it is still transcribed within the host cell. Initially, there were two major approaches that were frequently used to achieve a transient expression of a heterologous protein in plants: via viral vectors or via Agrobacterium. Generally, these approaches allow the production of large amounts of recombinant proteins in short periods of time. The viral vector approach profits from the capacity of viruses to infect the plant by moving short and long distances producing a systemic infection. The translational fusion of a small peptide to a viral protein has biological application since recombinant viruses can be isolated and then used as epitope carriers for vaccine production $[27,28]$. Recombinant proteins can also be obtained as non-fusion proteins [21] (also see [29]). The Agrobacterium-based method, called "agroinfiltration", involves the injection or vacuum infiltration of whole plants or parts of them with a suspension of bacteria harboring the construct of interest. This approach has a wide spectrum of applications and has resulted useful for the study of glycosylation [30], protein-protein interaction [31], conformation, folding, virus-like particles formation [32] and to produce interesting molecules for the therapeutic industry as monoclonal antibodies [25, 33] and antigens of human pathogens [34].

Nowadays, a new transient expression strategy for heterologous proteins production in plants is being used to overcome the limitations possessed by early platforms. It combines the two technologies mentioned above: the agroinfiltration method and Tobacco Mosaic Virus (TMV)based viral vectors system. This new approach, termed Magnifection, was first developed by Icon Genetics (now part of Bayer CropSciences, Germany) and allows the scalable production of a desired protein with high expression level and yield, low up- and downstream costs, reduced time, and most of all, reduced biosafety concerns [35, 36].

Mett et al. (2008) have employed a similar approach, using a launch vector system, to produce a plant subunit vaccine with potential human application. The authors demonstrated that plant-derived antigens of influenza virus were highly immunogenic and protected ferrets against the viral challenge [34].

\section{MUCOSAL IMMUNITY}

Most of the available vaccines are aimed at stimulating the production of serum antibodies against disease causing agents. However, the natural transmission of several infectious pathogens occurs at the mucosal level, and the first target for those pathogens replication may be the mucosa-associated lymphoid tissue [37]; then, successfully induction of strong 
mucosal immunity may require oral or nasal immunization. Given the progressive understanding of the mucosal immune system, it is now plausible to believe that the current injectiontype vaccines could be advanced to needle/syringe-free and cold-chain free forms of mucosal vaccines [38]. In this context, transgenic plants are invaluable tools as bioreactors for the production of antigens. They offer several advantages over conventional production systems, already mentioned, and they can be used as vaccines themselves. One of the major challenges in the field is modulating immune responses against infectious agents at the mucosal level, and many researchers working on the development of vaccines based on transgenic plants are addressing this issue (for an example see [39]).

\section{ORAL TOLERANCE}

One issue that remains to be clarified is the propensity of an edible plant-based vaccine of provoking oral tolerance. In this context, the term tolerance refers to the suppression of the immune response to the same antigen whether oral or parenteral immunization is used. The biological mean of this mechanism is to prevent immune responses against food antigens or bacteria that reside as commensals in the gut mucosa, needed for digestion and absorption. Many factors determine the immunogenicity or tolerogenicity effects of foreign antigens, such as: amount, persistence, portal of entry, presence of adjuvants and properties of antigen-presenting cells, among others. Different doses of orally administered antigens may induce anergy in antigen specific $\mathrm{T}$ cells or may stimulate the production of cytokines such as TGF- $\beta$ that inhibits lymphocyte proliferation, resulting in the suppression of the immune response. In general, large doses and prolonged persistence of a soluble protein without adjuvanticity would induce oral tolerance.

One way of studying this phenomenon is by measuring the activation of regulatory $T$ cells (Tregs) concomitantly to the study of cellular and humoral immune responses to a specific antigen. Tregs are a subpopulation of CD4+ T cells that play a major role in controlling and suppressing other cellular functions. There are few reports where cellular immunity is studied after the administration of an edible vaccine. This fact maybe due to the main interest in inducing a humoral response (systemic or secretory antibodies such as sIgA) with a plant based vaccine. In the same way, just two reports attempted to study tolerance in mice elicited by oral immunization of transgenic plants [40, 41]. Until now, the most conclusive results were that mice fed with transgenic tobacco expressing low content of antigen ( $0.5 \mathrm{ng}$ of HBsAg in $5 \mu \mathrm{g}$ of tissue) after a DNA prime injection, showed an anti- HBsAg humoral immune response detected in the GALT ( $\operatorname{IgA}$ in feces) and in the periphery (IgA and IgG in serum), which correlated with low Treg titers, while higher doses of HBsAg and plant tissue positively correlated with increases in Tregs. These results suggested the presence of oral tolerance avoiding the immune response to the tobacco plants. By adjusting the antigen dose to minimize the negative effects of tolerance, the immune response could be improved [41].

In the opposite perspective, transgenic plants were used to induce tolerance. Transgenic rice seeds expressing T-cell epitopes from cedar pollens allergens were fed to mice in order to prevent allergen specific response [42]. Results showed a significant decrease of serum $\operatorname{IgG}$ and $\operatorname{IgE}$ in mice fed with transgenic rice compared to control groups $(p<0.01)$, a significant inhibition of allergen-induced Th2 cytokines (IL4, IL-5, IL-13) measured in culture supernatants of stimulated$\mathrm{CD}+4 \mathrm{~T}$ cells, and a significant inhibition of histamine release in serum. It was also observed that after intranasal challenge with pollen proteins mice presented a reduced number of sneezing and pollinosis symptoms. This way, it was demonstrated that treatment with transgenic rice expressing allergen T-cell epitopes is effective in producing a state of desensitization or tolerance. In consequence, plants could be used in immunotherapy.

Several researchers have contributed in an encouraging way to the knowledge of the immune response elicited by plant-based vaccines whether in mice or in humans. However, much less is known about the tolerogenicity effects of an oral plant-based vaccine. In consequence, it is worthy to extend the resources to the study of this phenomenon onward the obtainment of a cost-effective vaccine.

\section{ADVANCES MADE TOWARD PLANT-BASED VACCINES AGAINST MAJOR HUMAN ILLNESS}

\subsection{Respiratory Diseases}

\subsubsection{Tuberculosis}

Tuberculosis (TB) is an infectious bacterial disease caused by Mycobacterium tuberculosis, a pathogen which affects the lungs. The disease is endemic in poor countries and it is related to poverty; nevertheless, the TB incidence is also increasing in industrialized countries. Improved TB vaccines are widely considered as a key element for successful TB control, and the development of efficient, safe and affordable vaccines against TB must remain a global priority.

The bacillus Calmette-Guérin (BCG) is the only available vaccine against this disease. It is derived from Mycobacterium bovis and induces high levels of protection in animal models but its efficacy in humans is highly variable [43]. One of the main antigen of Mycobacterium tuberculosis that is being targeted for the development of new subunit vaccines is ESAT 6 , a $6 \mathrm{kDa}$ secretory protein that induces a potent cellular response and that it is absent in $M$ bovis BCG [44]. This protein was successfully expressed in Arabidopsis thaliana as a fusion protein with the enterotoxigenic Escherichia coli heat- labile toxin B subunit (LTB) and proved to be immunogenic when orally administered to mice, since a specific Th1 response was observed $[45,46]$. ESAT 6 was also expressed in Nicotiana benthamiana by the Agrobacterium-mediated delivery of TMV-based vectors along with Ag85B, other TB antigen [47]. It was also expressed in Nicotiana tabacum using a potato virus $\mathrm{X}$ (PVX)-derived vector as a fusion protein with the capsid protein (CP) [48]. Neither of these two last transiently transformed plants was evaluated as immunogen in animal models.

\subsubsection{Influenza}

Influenza is a highly contagious viral disease that typically results in fever and respiratory symptoms with frequent complications that can eventually lead to hospitalization and death, particularly in young children, adults over 65 , and individuals with certain chronic underlying health conditions 
[49]. Antibodies to influenza viral proteins hemagglutinin (HA) and neuraminidase (NA) play a key role in the protective efficacy of influenza vaccines [50]. Thus, HA and NA are supposed to be the best candidates for influenza subunit vaccine development. Mett et al. (2008) transiently expressed the stem and globular domains of HA in Nicotiana benthamiana plants, as fusions to a carrier protein [34]. The authors immunized ferrets with purified antigens. These plantproduced antigens were highly immunogenic and conferred complete protection against infection in the ferret challenge model. When they added the plant-produced NA, an enhancement of the immune response was observed [34]. Virus like particles (VLPs) were also obtained in transiently transformed plants and assayed as potential immunogens against influenza. Preclinical trials with influenza VLPs have demonstrated their capacity to induce both humoral and cellular immune responses at low antigen doses [51]. As they are composed of noninfectious recombinant proteins, the production of VLPs does not require, as any recombinant protein production methodology, the protective measures imposed by the manipulation of highly infectious pandemic virus particles. However, influenza VLPs production currently relies on the use of cell culture systems that may not meet the cost and speed constraints posed by global pandemic scenarios. An alternative method for the production of influenza VLPs at lower costs was developed by D'Aoust $e t$ al. (2008) utilizing transgenic plants. The authors demonstrated that influenza VLPs can form and accumulate in agroinfiltrated Nicotiana benthamiana leaves producing HA from either $\mathrm{A} /$ Indonesia/05/05 (H5N1) or A/New Caledonia/20/99 (H1N1). Furthermore, they showed that the parenteral administration of purified H5-VLPs with alum induces a strong and protective immune response in mice [52].

Highly pathogenic $\mathrm{H} 5 \mathrm{~N} 1$ avian influenza continues to be a human health threatens, with mortality rates exceeding $60 \%$. The risk of an $\mathrm{H} 5 \mathrm{~N} 1$ pandemic has encouraged global efforts to develop vaccines against highly pathogenic avian influenza (HPAI). Shoji et al. (2009) recently described the production of HA from the A/Indonesia/05/05 strain of H5N1 influenza virus by transient expression in Nicotiana benthamiana plants. They demonstrated that immunization of mice and ferrets with plant-derived HA elicited serum hemagglutinin-inhibiting antibodies and protected ferrets against challenge infection with a homologous virus [53].

\subsection{Diarrheal Diseases}

The gastrointestinal infection by some bacteria, virus and parasitic organisms usually produced diarrhea, a lifethreatening to young children, malnourished or immune compromised persons. The principal sources of infection are contaminated food or drinking-water and person to person contagious due to poor hygiene. Pathogens infecting the gastroenteric tract have been targeted for the development of oral plant-based vaccines.

\subsubsection{Bacterial Diarrheas}

Enterotoxigenic Escherichia coli (ETEC) is a common cause of infectious diarrhea in tropical climates where uncontaminated drinking water is not readily available, being the infants the most affected. The E. coli heat-labile enterotoxin (LT) is the major ETEC pathogenic factor, however the LT subunit B (LT-B) is immunogenic, possesses adjuvant activity and lacks toxicity, what make it a viable antigen to vaccinate against ETEC diarrhea [54]. ETEC diarrhea has much in common with cholera. Both diseases result from the ingestion of rather large inocula of gram negative bacteria that colonize the small intestine and produce toxins causing net secretion of fluid into the intestinal lumen [55]. LT structure and function are similar to those of cholera toxin (CT): both contain A and B subunits whose amino acid sequences show $80 \%$ identity [56]. Due to this homology, immunization with LTB raises an antitoxin response that extends to the CTB subunit, thereby providing equally strong protection against oral LT or CT challenge [57].

In 1995, Haq and colleagues expressed the B subunit of the heat-labile enterotoxin (LT-B) from E. coli in potato and tobacco plants for the first time. They observed that the LT-B could assemble into pentameric structures retaining the ganglioside binding properties like bacteria-derived LT-B. Mice fed with transgenic tubers developed a specific immune response demonstrated by the presence of serum $\operatorname{IgG}$ and mucosal IgA production [17]. In a subsequent study, mice fed three weekly doses of tubers containing either 20 or $50 \mu \mathrm{g}$ of LT-B developed high levels of specific immunoglobulins and when challenged with $25 \mu \mathrm{g}$ of LT orally administered, a reduction in the gut/carcass mass ratios was observed. However, none of the vaccinated animals were completely protected [58]. Results obtained in the animal model led to studies in humans. Eleven volunteers ingested three oral doses of either $100 \mathrm{~g}$ or $50 \mathrm{~g}$ of raw potato containing approximately $0.4-1.1 \mathrm{mg}$ LT-B and three other volunteers ingested $50 \mathrm{~g}$ of non-transgenic potato. The results showed an increase of at least four-fold for IgG and secretory IgA anit-LT-B after immunization [59]. Some years later, another clinical trial was conducted in human volunteers to test transgenic corn immunogenicity. Results showed specific antibodies production against LT-B as a consequence of oral immunization by corn feeding [60].

LT-B expression, characterization and immunological capabilities in mice were also studied using other plant species such as tomato [61], tobacco (chloroplast) [62], Nicotiana benthamiana (TMV-based viral vector) [63] and soybean [64] with relative success. In turn, potato-expressed Cholera toxin $B$ subunit (CTB) has also been found to induce a protective immune response in mice [15].

\subsubsection{Rotavirus}

Rotavirus is one of the most common causes of diarrhea, and severe infection (rotavirus gastroenteritis) is the leading cause of acute, dehydrating diarrhea in infants and young children. Diarrhea caused by rotavirus is responsible for more than half a million deaths worldwide every year. This is especially true in developing countries, where nutrition and health care are not optimal. The transmission of the viral agent is by the fecal-oral route. The virus infects cells that line the small intestine and produces an enterotoxin, which induces gastroenteritis, leading to severe diarrhea and sometimes death through dehydration. Several developments on plant-based vaccines against this agent have been reported. A codonoptimized gene (sVP6) encoding the VP6 protein of human group A rotavirus was synthesized and inserted into the alfalfa genome using agrobacterium-mediated transformation [65]. Female BALB/c mice were gavaged weekly with $10 \mathrm{mg}$ of transgenic alfalfa extract $\mathrm{CpG}$-rich oligodeoxynucleotides as 
mucosal adjuvant and immunized animals developed high titers of anti-VP6 serum IgG and mucosal IgA. Moreover, their offspring developed less severe diarrhea after challenge with simian rotavirus SA-11, indicating that antibodies generated in the dams provided passive protection to the newborn mice [65].

Rotavirus protein VP7 was also tested using plant expression systems. Wu et al. (2003) found that rotavirus VP7 could maintain its neutralizing immunity when it was expressed in transgenic potato plants. Mice immunized with the transformed tubers successfully elicited serum IgG and mucosal IgA [66]. Later on, the same group of researchers demonstrated that humoral and mucosal immune responses were successfully induced in BALB/c mice fed with the fiftieth generation of the transformed potato tubers [67].

Another approach was reported by Saldaña et al. (2006). In this case the successful expression of rotavirus capsid proteins VP2 and VP6 in fruits of transgenic tomato plants was demonstrated. The authors were able to recover rotavirus VLPs from tomato fruit, even though only a small proportion of VP2/VP6 assembled into VLPs. To investigate immunogenicity, adult mice were immunized intraperitoneally three times with a protein extract from transgenic tomatoes in adjuvant. Transgenic tomato extract induced detectable levels of anti-rotavirus antibodies in serum; however, the contribution of either the free rotavirus proteins or the VLPs to the induction of the antibody response was not determined [68].

\subsubsection{Norwalk Virus}

Another important agent related to enteric diseases is a norovirus. Norwalk virus is a common cause of vomiting and diarrheal illness each winter and has often been referred to as "stomach flu". Norwalk and Noroviruses are transmitted primarily by the fecal-oral route. The disease is transmitted through ingestion of contaminated foods and drinks, or by contact with an infected individual. Plant-based immunogens have been developed against this virus.

A human clinical trial was conducted after the demonstration that capsid proteins of Norwalk virus assembled into virus like particles in potato and tobacco plants retaining the immunological properties of wild type virus. Thus, in 2000, Tacket and coworkers carried out a study with 24 volunteers, 10 receiving 2 doses of transgenic potato at days 0 and 21, and a dose of non transgenic potato on day 7; other 10 receiving 3 doses at 0,7 and 21 days; and 4 receiving non transgenic potato on 0,7 and 21 days. All volunteers ingested $150 \mathrm{~g}$ of raw, peeled, potato cubes containing a variable amount of protein, between 215 and $751 \mu \mathrm{g}$ of NVCP. The proportion of protein assembled into VLPs varied between $25-50 \%$. Four volunteers developed an increase in serum IgG antibody titers, 4 showed an increase in serum IgM (3 of whom did not develop IgG response) and 6 presented increased $\operatorname{IgA}$ in stool samples. Although the serum antibody response was modest, 19 out of 20 individuals showed significant increases of IgA antibody-forming cells [69].

\subsection{Sexually Transmitted Diseases}

Viruses responsible of sexually transmitted diseases were also addressed through the development of plant-based vaccine field. In this regard, some key antigens of human immunodeficiency virus (HIV), hepatitis B virus (HBV) and papillomavirus (HPV) have been expressed in planta arriving in some cases to phase I clinical trials.

\subsubsection{Human Papilloma}

One example of the progress made in this field is the one concerning HPV. Maclean et al. (2007) have reported the expression of L1 capsid protein from HPV-16. By a transient expression assay, the authors determined not only that L1 protein was capable of assemble into VLPs, maintaining its immunological properties, but that a human instead of a plant codon usage and the protein-direction to the chloroplast, were the best conditions to achieve high yields of recombinant L1 protein (11\% of TSP in transgenic tobacco) [70]. These results showed an efficacious way of producing VLPs onward HPV vaccine, maybe a cheaper one than the new HPV vaccine produced in insect cells. Immunizing experiments are needed to better prove this hypothesis.

\subsubsection{HIV/AIDS}

Studies done on plant-expressed HIV proteins have largely focused on HIV-1 relevant epitopes belonging to the regulatory protein (Tat), p24 core protein, nef protein or envelope glycoproteins (gp120 and gp41). In some of these studies, immunological properties of plant derived HIVantigens have been proved in mice [71-74] or rabbit [75]. As the attention is generally focused on obtaining acceptable levels of the recombinant protein to develop a viable vaccine, Obregon and coworkers showed an improved stability of p24 core protein by fusing it to the constant domains ( $\alpha 2$ and $\alpha 3$ ) of human IgA heavy chain. The fusion protein $\mathrm{p} 24 / \alpha 2-\alpha 3$ rendered more protein than $\mathrm{p} 24$ alone (13-fold) as it could be measured by ELISA and western blot assay. Fusion protein formed dimmers that were retained within the cell resulting in an enhanced expression, apparently related to protein folding processing and assembly, subcellular targeting and protein stability [76].

\subsubsection{Hepatitis $B$}

Infection by HBV is the main cause of liver cancer. It can provoke cirrhosis and liver insufficiency. By means of genetic engineering techniques, three subunit vaccines have been developed based on the purified Hepatitis B virus surface antigen (HBsAg). They are produced in yeast cells (Recombivax $\mathrm{HB}$ and Engerix-B) and in mammalian cell cultures (GenHevac B) and proved to be safe, immunogenic and efficacious in preventing the HBV infection. Interesting attempts were made in the plant-expression field to develop an edible plant-based vaccine capable of eliciting a mucosal immune response. Several edible plant species like banana, lettuce, lupin, tomato and potato were employed as hosts for the expression of HBsAg and several approaches in animal model resulted successful. Encouraged by previous results, Kapusta and coworkers performed the first, although small, study in humans (Thomas Jefferson University, USA). Volunteers who ate raw transgenic lettuce leaves developed specific-IgG response. Two out of the three human volunteers mounted a significant immune response after a second ingestion of transgenic lettuce [77]. Then, in 2005, Thanavala and coworkers carried out a clinical trial with previously vaccinated individuals. Volunteers were fed at 2-weeks interval with $100 \mathrm{~g}$ of uncooked potato containing $8.5 \mu \mathrm{g}$ of 
HBsAg per gram of transgenic potato. $63 \%(10 / 16)$ of individuals who ate 3 doses and $53 \%(9 / 17)$ of those who ate 2 doses exhibited marked increases in serum antibody titers compared with the pre feeding measures, with boosting effects and without the addition of any mucosal adjuvant. The results obtained in this study demonstrated that an orally administered plant-based vaccine could be successful in promoting an immune response. The authors proposed this type of vaccine as a tool to be included during a global immunization program [78].

\subsection{Other Diseases of Concern in Developing Countries}

\subsubsection{Dengue Fever}

Dengue virus is a mosquito-borne human pathogen and currently the most important flavivirus causing human disease in the tropical and subtropical regions of the world. Four antigenically related serotypes of dengue virus (dengue 1, 2, 3 and 4 viruses) induce a spectrum of diseases ranging from a simple febrile illness, dengue fever, to a fatal dengue hemorrhagic fever/dengue hemorrhagic shock syndrome [79].

As there is no cross-protection between the four serotypes, it is very difficult to develop an efficient vaccine against dengue. Moreover, progress in this field has been very low because these viruses grow poorly in cell culture (www.who.int/vaccines-documents/). Different strategies have been attempted to develop vaccine candidates: attenuated, recombinant, subunit, chimeric and DNA vaccines. Most of the recombinant DNA-based strategies have focused on the envelope (E) protein, responsible for the induction of neutralizing antibodies [80]. Recently, Saejung and coworkers reported, for the first time, the production of a dengue vaccine in plants. They expressed a gene fragment coding for domain III of dengue 2 envelope protein in Nicotiana benthamiana plants using a tobacco mosaic virus (TMV)-based transient expression system. When the recombinant protein was evaluated in mice i.m. immunized, it induced the production of neutralizing antibodies. This constitutes a promising result, although further studies in order to improve the immunological properties of the recombinant protein are needed, as the antibody titers obtained were low [81].

\subsubsection{Rabies}

Rabies is a viral disease of mammals and is most commonly transmitted through the bite of a rabid animal. Globally, an estimated of 10 million people receive post exposure antiserum treatment each year, after being exposed to rabies-suspect animal (WHO Fact Sheet number 99, 2001). Modern vaccines for humans are expensive, especially for densely populated countries in Africa and Asia, where rabies is endemic and remains a major health problem. The rabies virus glycoprotein ( $G$ protein) is the major viral protein responsible for the induction of a protective immune response, while the nucleoprotein ( $\mathrm{N}$ protein) triggers $\mathrm{T}$ cell responses, facilitating the production of neutralizing antibodies and other immune functions [82].

In the field of plant-based vaccines, different approaches have been made in order to obtain a rabies vaccine that could be a low cost alternative to orally immunize children and older persons of developing countries where rabies is highly endemic. In 1997, Yusibov and collaborators expressed chimeric antigenic peptides from $\mathrm{G}$ and $\mathrm{N}$ proteins of rabies in tobacco. Purified virus particles from the infected tobacco plant tissue were used to immunize mice. Mice received intraperitoneally 7 doses $(10 \mu \mathrm{g} / \mathrm{dose})$ of recombinant virus at 2 -week intervals resulting in the production of specific antibodies capable of neutralizing a CVS-11 strain rabies virus. These results showed that rabies antigen produced by the recombinant viral approach was able to elicit a protective response against rabies [71]. Then, it was demonstrated by a similar approach that mice intraperitoneally or orally inoculated with virus-infected spinach leaves mounted a local and systemic immune response [83]. Later on, the group conducted a trial with human volunteers. One group of five individuals, previously immunized against rabies virus, was fed three times with $20 \mathrm{~g}$ of uncooked spinach leaves containing recombinant virus ( $84 \mu \mathrm{g}$ of chimeric peptides each dose). The other group of nine rabies-naïve subjects ate three doses of $150 \mathrm{~g}$ of raw spinach containing $700 \mu \mathrm{g}$ of rabies antigens per dose. Significantly increases in specific serum $\operatorname{IgG}$ were obtained for 3 out of 5 individuals of this group, while 5 out of 9 naïve individuals presented low increases of serum specific antibody, suggesting the potential used of plant based vaccines as a supplementary oral booster for human rabies vaccination [84]. Finally, Loza-Rubio and coworkers developed transgenic maize expressing the rabies virus glycoprotein of the Vnukovo strain and they evaluated its immunogenicity in mice by the oral route. Animals were fed once with $50 \mu \mathrm{g}$ of protein and challenged 90-days later with a rabies virus isolated from vampire bats. The edible vaccine induced viral neutralizing antibodies which protected mice $100 \%$ against challenge [85].

\section{CONCLUDING REMARKS}

The use of plant-based systems for producing vaccine antigens would offer many practical advantages that makes them an attractive option and a practical methodology for the propagation of mucosal vaccines on a global scale $[10,86]$ : it would be less expensive than to produce antigens via industrial fermentation; there would be no need to purify the antigen if it is expressed in plant edible tissue; the plant expression system minimizes risks arising from contamination.

As a first step towards a potential plant-based mucosal vaccine, Curtiss and Cardineau expressed the surface protein antigen of Streptcoccus mutans, the causative agent for dental caries, in tobacco [87]. Since then, as we mentioned before, many vaccine antigen candidates, have been expressed in different plant species to demonstrate the feasibility of oral plant-based vaccines $[15,17-19,58,59,88,89]$. However, these plant-based vaccines have not yet advanced from the experimental laboratory to a practical application.

We believe that there is a possible future for plant-based vaccines and that the benefits of this tool could be of great help in developing countries, where the need is often the greatest. However, different issues will have to be established and well defined first: good expression levels, product quality and doses, costs of downstream process, regulatory framework, efficacy and safety, and cooperation with the industry.

\section{REFERENCES}

[1] Watanabe I, Hagiwara Y, Kadowaki SE, et al. Characterization of protective immune responses induced by nasal influenza vaccine 
containing mutant cholera toxin as a safe adjuvant (CT112K). Vaccine 2002; 20(29-30): 3443-55.

[2] Bukreyev A, Lamirande EW, Buchholz UJ, et al. Mucosal immunisation of African green monkeys (Cercopithecus aethiops) with an attenuated parainfluenza virus expressing the SARS coronavirus spike protein for the prevention of SARS. Lancet 2004; 363(9427): 2122-7.

[3] Belyakov IM, Hel Z, Kelsall B, et al. Mucosal AIDS vaccine reduces disease and viral load in gut reservoir and blood after mucosal infection of macaques. Nat Med 2001; 7(12): 1320-6.

[4] Czerkinsky C, Anjuere F, McGhee JR, et al. Mucosal immunity and tolerance: relevance to vaccine development. Immunol Rev 1999; 170: $197-222$.

[5] Kiyono H, Fukuyama S. NALT- versus Peyer's-patch-mediated mucosal immunity. Nat Rev Immunol 2004; 4(9): 699-710.

[6] Yuki Y, Kiyono H. New generation of mucosal adjuvants for the induction of protective immunity. Rev Med Virol 2003; 13(5): 293 310 .

[7] Holmgren J, Czerkinsky C. Mucosal immunity and vaccines. Nat Med 2005; 11(4 Suppl): S45-53.

[8] Neutra MR, Kozlowski PA. Mucosal vaccines: the promise and the challenge. Nat Rev Immunol 2006; 6(2): 148-58.

[9] Chargelegue D, Obregon P, Drake PM. Transgenic plants for vaccine production: expectations and limitations. Trends Plant Sci 2001; 6(11): 495-6.

[10] Varmus H, Klausner R, Zerhouni E, et al. Public health. Grand Challenges in Global Health. Science 2003; 302(5644): 398-9.

[11] Levine MM, Sztein MB. Vaccine development strategies for improving immunization: the role of modern immunology. Nat Immunol 2004; 5(5): 460-4.

[12] Ryan EJ, Daly LM, Mills KH. Immunomodulators and delivery systems for vaccination by mucosal routes. Trends Biotechnol 2001; 19(8): 293-304.

[13] Fischer R, Emans N. Molecular farming of pharmaceutical proteins. Transgenic Res 2000; 9(4-5): 279-99; discussion 277.

[14] Fischer R, Hoffmann K, Schillberg S, et al. Antibody production by molecular farming in plants. J Biol Regul Homeost Agents 2000; 14(2): 83-92.

[15] Arakawa, Chong DK, Langridge WH. Efficacy of a food plant-based oral cholera toxin B subunit vaccine. Nat Biotechnol 1998; 16(3): 292-7.

[16] Berinstein A, Vazquez-Rovere C, Asurmendi S, et al. Mucosal and systemic immunization elicited by Newcastle disease virus (NDV) transgenic plants as antigens. Vaccine 2005; 23(48-49): 5583-9.

[17] Haq TA, Mason HS, Clements JD, et al. Oral immunization with a recombinant bacterial antigen produced in transgenic plants. Science 1995; 268(5211): 714-6.

[18] Mason HS, Ball JM, Shi JJ, et al. Expression of Norwalk virus capsid protein in transgenic tobacco and potato and its oral immunogenicity in mice. Proc Natl Acad Sci USA 1996; 93(11): 5335-40.

[19] Sandhu JS, Krasnyanski SF, Domier LL, et al. Oral immunization of mice with transgenic tomato fruit expressing respiratory syncytial virus-F protein induces a systemic immune response. Transgenic Res 2000; 9(2): 127-35.

[20] Thanavala Y, Yang YF, Lyons P, et al. Immunogenicity of transgenic plant-derived hepatitis B surface antigen. Proc Natl Acad Sci USA 1995; 92(8): 3358-61.

[21] Wigdorovitz A, Pérez Filgueira DM, Robertson N, et al. Protection of mice against challenge with foot and mouth disease virus (FMDV) by immunization with foliar extracts from plants infected with recombinant tobacco mosaic virus expressing the FMDV structural protein VP1. Virology 1999; 264(1): 85-91.

[22] Galeffi P, Lombardi A, Donato MD, et al. Expression of single-chain antibodies in transgenic plants. Vaccine 2005; 23(15): 1823-7.

[23] Ma JK, Hiatt A, Hein M, et al. Generation and assembly of secretory antibodies in plants. Science 1995; 268(5211): 716-9.

[24] Ma JK, Hikmat BY, Wycoff K, et al. Characterization of a recombinant plant monoclonal secretory antibody and preventive immunotherapy in humans. Nat Med 1998; 4(5): 601-6.

[25] Villani ME, Morgun B, Brunetti P, et al. Plant pharming of a fullsized, tumour-targeting antibody using different expression strategies. Plant Biotechnol J 2009; 7(1): 59-72.

[26] De Cosa B, Moar W, Lee SB, et al. Overexpression of the Bt cry $2 \mathrm{Aa} 2$ operon in chloroplasts leads to formation of insecticidal crystals. Nat Biotechnol 2001; 19(1): 71-4.
[27] Natilla A, Piazzolla G, Nuzzaci M, et al. Cucumber mosaic virus as carrier of a hepatitis C virus-derived epitope. Arch Virol 2004; 149(1): 137-54.

[28] Natilla A, Hammond RW, Nemchinov LG. Epitope presentation system based on cucumber mosaic virus coat protein expressed from a potato virus X-based vector. Arch Virol 2006; 151(7): 1373-86.

[29] Pogue GP, Lindbo JA, Garger SJ, et al. Making an ally from an enemy: plant virology and the new agriculture. Annu Rev Phytopathol 2002; 40: 45-74.

[30] van der Hoorn RA, Wulff BB, Rivas S, et al. Structure-function analysis of cf-9, a receptor-like protein with extracytoplasmic leucine-rich repeats. Plant Cell 2005; 17(3): 1000-15.

[31] Citovsky V, Lee LY, Vyas S, et al. Subcellular localization of interacting proteins by bimolecular fluorescence complementation in planta. J Mol Biol 2006; 362(5): 1120-31.

[32] Huang Z, Mason HS. Conformational analysis of hepatitis B surface antigen fusions in an Agrobacterium-mediated transient expression system. Plant Biotechnol J 2004; 2 (3): 241-9.

[33] Orzaez D, Mirabel S, Wieland WH, et al. Agroinjection of tomato fruits. A tool for rapid functional analysis of transgenes directly in fruit. Plant Physiol 2006; 140(1): 3-11.

[34] Mett V, Musiychuk K, Bi H, et al. A plant-produced influenza subunit vaccine protects ferrets against virus challenge. Influenza and Other Respiratory Viruses 2008; 2(1): 33-40.

[35] Gleba Y, Klimyuk V, Marillonnet S. Magnifection--a new platform for expressing recombinant vaccines in plants. Vaccine 2005; 23(1718): 2042-8.

[36] Marillonnet S, Thoeringer C, Kandzia R, et al. Systemic Agrobacterium tumefaciens-mediated transfection of viral replicons for efficient transient expression in plants. Nat Biotechnol 2005; 23(6): 718-23.

[37] Veazey RS, DeMaria M, Chalifoux LV, et al. Gastrointestinal tract as a major site of CD4+ T cell depletion and viral replication in SIV infection. Science 1998; 280(5362): 427-31.

[38] Mestecky J, Nguyen H, Czerkinsky C, et al. Oral immunization: an update. Curr Opin Gastroenterol 2008; 24(6): 713-9.

[39] Takahashi I, Nochi T, Yuki Y, et al. New horizon of mucosal immunity and vaccines. Curr Opin Immunol 2009; 21(3): 352-8.

[40] Guetard D, Greco R, Cervantes Gonzalez M, et al. Immunogenicity and tolerance following $\mathrm{HIV}-1 / \mathrm{HBV}$ plant-based oral vaccine administration. Vaccine 2008; 26(35): 4477-85.

[41] Kostrzak A, Cervantes Gonzalez M, Guetard D, et al. Oral administration of low doses of plant-based HBsAg induced antigenspecific IgAs and IgGs in mice, without increasing levels of regulatory T cells. Vaccine 2009; 27(35): 4798-807.

[42] Takagi H, Hiroi $\mathrm{T}$, Yang L, et al. A rice-based edible vaccine expressing multiple $\mathrm{T}$ cell epitopes induces oral tolerance for inhibition of Th2-mediated IgE responses. Proc Natl Acad Sci USA 2005; 102(48): 17525-30.

[43] Fine PE. Variation in protection by BCG: implications of and for heterologous immunity. Lancet 1995; 346(8986): 1339-45.

[44] Harboe M, Oettinger T, Wiker HG, et al. Evidence for occurrence of the ESAT-6 protein in Mycobacterium tuberculosis and virulent Mycobacterium bovis and for its absence in Mycobacterium bovis BCG. Infect Immun 1996; 64(1): 16-22.

[45] Rigano MM, Alvarez ML, Pinkhasov J, et al. Production of a fusion protein consisting of the enterotoxigenic Escherichia coli heat-labile toxin B subunit and a tuberculosis antigen in Arabidopsis thaliana. Plant Cell Rep 2004; 22(7): 502-8.

[46] Rigano MM, Dreitz S, Kipnis AP, et al. Oral immunogenicity of a plant-made, subunit, tuberculosis vaccine. Vaccine 2006; 24(5): 6915.

[47] Dorokhov YL, Sheveleva AA, Frolova OY, et al. Superexpression of tuberculosis antigens in plant leaves. Tuberculosis (Edinb) 2007; 87(3): 218-24.

[48] Zelada AM, Calamante G, de la Paz Santangelo M, et al. Expression of tuberculosis antigen ESAT-6 in Nicotiana tabacum using a potato virus X-based vector. Tuberculosis (Edinb) 2006; 86(3-4): 263-7.

[49] Stuart-Harris CH, Schild GC, Oxford JS. Influenza: The Viruses and the Disease. London: Edward Arnold 1985.

[50] Brown F, Haaheim LR, Wood JM, et al. Eds. Laboratory Correlates of Immunity to Influenza - A Reassessment. Basel: Karger 2002.

[51] Bright RA, Carter DM, Daniluk S, et al. Influenza virus-like particles elicit broader immune responses than whole virion inactivated influenza virus or recombinant hemagglutinin. Vaccine 2007; 25(19): $3871-8$. 
[52] D'Aoust MA, Lavoie PO, Couture MM, et al. Influenza virus-like particles produced by transient expression in Nicotiana benthamiana induce a protective immune response against a lethal viral challenge in mice. Plant Biotechnol J 2008; 6(9): 930-40.

[53] Shoji Y, Bi H, Musiychuk K, et al. Plant-derived hemagglutinin protects ferrets against challenge infection with the $\mathrm{A} /$ Indonesia/05/05 strain of avian influenza. Vaccine 2009; 27(7): 1087-92.

[54] Belisl, BW, Twiddy EM, Holmes RK. Monoclonal antibodies with an expanded repertoire of specificities and potent neutralizing activity for Escherichia coli heat-labile enterotoxin. Infect Immun 1984; 46(3): 759-64.

[55] Wolf MK. Occurrence, distribution, and associations of $\mathrm{O}$ and $\mathrm{H}$ serogroups, colonization factor antigens, and toxins of enterotoxigenic Escherichia coli. Clin Microbiol Rev 1997; 10(4): $569-84$.

[56] Sixma TK, Pronk SE, Kalk KH, et al. Crystal structure of a cholera toxin-related heat-labile enterotoxin from E. coli. Nature 1991; 351(6325): 371-7

[57] Klipstein FA, Engert RF, Clements JD, Houghten RA. Differences in cross-protection in rats immunized with the B subunits of cholera toxin and Escherichia coli heat-labile toxin. Infect Immun 1984; 43(3): 811-6.

[58] Mason HS, Haq TA, Clements JD, et al. Edible vaccine protects mice against Escherichia coli heat-labile enterotoxin (LT): potatoes expressing a synthetic LT-B gene. Vaccine 1998; 16(13): 1336-43.

[59] Tacket CO, Mason HS, Losonsky G, et al. Immunogenicity in humans of a recombinant bacterial antigen delivered in a transgenic potato. Nat Med 1998; 4(5): 607-9.

[60] Tacket CO, Pasetti MF, Edelman R, et al. Immunogenicity of recombinant LT-B delivered orally to humans in transgenic corn. Vaccine 2004; 22(31-32): 4385-9.

[61] Walmsley AM, Alvarez ML, Jin Y, et al. Expression of the B subunit of Escherichia coli heat-labile enterotoxin as a fusion protein in transgenic tomato. Plant Cell Rep 2003; 21(10): 1020-6.

[62] Kang TJ, Loc NH, Jang MO, et al. Expression of the B subunit of E. coli heat-labile enterotoxin in the chloroplasts of plants and its characterization. Transgenic Res 2003; 12(6): 683-91.

[63] Wagner B, Hufnagl K, Radauer C, et al. Expression of the B subunit of the heat-labile enterotoxin of Escherichia coli in tobacco mosaic virus-infected Nicotiana benthamiana plants and its characterization as mucosal immunogen and adjuvant. J Immunol Methods 2004; 287(1-2): 203-15.

[64] Moravec T, Schmidt MA, Herman EM, et al. Production of Escherichia coli heat labile toxin (LT) B subunit in soybean seed and analysis of its immunogenicity as an oral vaccine. Vaccine 2007; 25(9): 1647-57.

[65] Dong JL, Liang BG, Jin YS, et al. Oral immunization with pBsVP6transgenic alfalfa protects mice against rotavirus infection. Virology 2005;339(2): 153-63.

[66] Wu YZ, Li JT, Mou ZR, et al. Oral immunization with rotavirus VP7 expressed in transgenic potatoes induced high titers of mucosal neutralizing IgA. Virology 2003; 313(2): 337-42.

[67] Li JT, Fei L, Mou ZR, et al. Immunogenicity of a plant-derived edible rotavirus subunit vaccine transformed over fifty generations. Virology 2006; 356(1-2): 171-8.

[68] Saldaña S, Esquivel Guadarrama F, Olivera Flores Tde J, et al. Production of rotavirus-like particles in tomato (Lycopersicon esculentum L.) fruit by expression of capsid proteins VP2 and VP6 and immunological studies. Viral Immunol 2006; 19(1): 42-53.

[69] Tacket CO, Mason HS, Losonsky G, et al. Human immune responses to a novel norwalk virus vaccine delivered in transgenic potatoes. $\mathrm{J}$ Infect Dis 2000; 182(1): 302-5.
[70] Maclean J, Koekemoer M, Olivier AJ, et al. Optimization of human papillomavirus type 16 (HPV-16) L1 expression in plants: comparison of the suitability of different HPV-16 L1 gene variants and different cell-compartment localization. J Gen Virol 2007; 88(Pt 5): 1460-9.

[71] Yusibov V, Modelska A, Steplewski K, et al. Antigens produced in plants by infection with chimeric plant viruses immunize against rabies virus and HIV-1. Proc Natl Acad Sci USA 1997; 94(11): 57848.

[72] Durrani Z, McInerney TL, McLain L, et al. Intranasal immunization with a plant virus expressing a peptide from HIV-1 gp41 stimulates better mucosal and systemic HIV-1-specific IgA and IgG than oral immunization. J Immunol Methods 1998; 220(1-2): 93-103.

[73] Marusic C, Rizza P, Lattanzi L, et al. Chimeric plant virus particles as immunogens for inducing murine and human immune responses against human immunodeficiency virus type 1. J Virol 2001; 75(18): 8434-9.

[74] Karasev AV, Foulke S, Wellens C, et al. Plant based HIV-1 vaccine candidate: Tat protein produced in spinach. Vaccine 2005; 23(15): 1875-80.

[75] Porta C, Spall VE, Loveland J, et al. Development of cowpea mosaic virus as a high-yielding system for the presentation of foreign peptides. Virology 1994; 202(2): 949-55.

[76] Obregon P, Chargelegue D, Drake PM, et al. HIV-1 p24immunoglobulin fusion molecule: a new strategy for plant-based protein production. Plant Biotechnol J 2006; 4(2): 195-207.

[77] Kapusta J, Modelska A, Figlerowicz M, et al. A plant-derived edible vaccine against hepatitis B virus. FASEB J 1999; 13(13): 1796-9.

[78] Thanavala Y, Mahoney M, Pal S, et al. Immunogenicity in humans of an edible vaccine for hepatitis B. Proc Natl Acad Sci USA 2005; 102(9): 3378-82.

[79] Konishi E, Yamaoka M, Kurane I, et al. A DNA vaccine expressing dengue type 2 virus premembrane and envelope genes induces neutralizing antibody and memory B cells in mice. Vaccine 2000; 18(11-12): 1133-9.

[80] Chang GJ. Molecular biology of dengue viruses, in Dengue and Dengue Hemorrhagic fever. 1997, the University Press: Cambridge; pp. 175-198.

[81] Saejung W, Fujiyama K, Takasaki T, et al. Production of dengue 2 envelope domain III in plant using TMV-based vector system. Vaccine $2007 ;$ 25(36): 6646-54.

[82] Tollis M, Dietzschold B, Volia CB, et al. Immunization of monkeys with rabies ribonucleoprotein (RNP) confers protective immunity against rabies. Vaccine 1991; 9(2): 134-6.

[83] Modelska A, Dietzschold B, Sleysh N, et al. Immunization against rabies with plant-derived antigen. Proc Natl Acad Sci USA 1998; 95(5): 2481-5.

[84] Yusibov V, Hooper DC, Spitsin SV, et al. Expression in plants and immunogenicity of plant virus-based experimental rabies vaccine. Vaccine 2002; 20(25-26): 3155-64

[85] Loza-Rubio E, Rojas E, Gómez L, et al. Development of an edible rabies vaccine in maize using the Vnukovo strain. Dev Biol (Basel) 2008; 131: 477-82.

[86] Streatfield SJ, Howard JA. Plant-based vaccines. Int J Parasitol 2003 33(5-6): 479-93.

[87] Curtis R, Cardineau G. Oral immunization by transgenic plants. in World patent application. USA: Washington University 1990.

[88] Richter LJ, Thanavala Y, Arntzen CJ, et al. Production of hepatitis B surface antigen in transgenic plants for oral immunization. Nat Biotechnol 2000; 18(11): 1167-71.

[89] Streatfield SJ, Jilka JM, Hood EE, et al. Plant-based vaccines: unique advantages. Vaccine 2001; 19(17-19): 2742-8. 